\title{
Norois
}

Environnement, aménagement, société

$194 \mid 2005 / 1$

Les milieux polaires et subpolaires de l'Atlantique

\section{Typologie des lacs polaires}

Une démarche de géographie limnologique

Classification of polar lakes: a geographical and limnological approach

\section{Laurent Touchart}

\section{(2) OpenEdition}

Journals

Édition électronique

URL : https://journals.openedition.org/norois/665

DOI : $10.4000 /$ norois. 665

ISBN : 978-2-7535-1542-0

ISSN : $1760-8546$

\section{Éditeur}

Presses universitaires de Rennes

\section{Édition imprimée}

Date de publication : 1 mars 2005

Pagination : $97-107$

ISBN : 978-2-7535-0100-3

ISSN : 0029-182X

\section{Référence électronique}

Laurent Touchart, «Typologie des lacs polaires », Norois [En ligne], 194 | 2005/1, mis en ligne le 13 août 2008, consulté le 13 janvier 2022. URL : http://journals.openedition.org/norois/665 ; DOl : https:// doi.org/10.4000/norois.665

Ce document a été généré automatiquement le 13 janvier 2022.

(c) Tous droits réservés 


\title{
Typologie des lacs polaires
}

\author{
Une démarche de géographie limnologique \\ Classification of polar lakes: a geographical and limnological approach
}

\author{
Laurent Touchart
}

\section{NOTE DE L'ÉDITEUR}

Cet article a été reçu le 6 septembre 2004 et définitivement accepté le 21 mars 2005.

1 La limnologie polaire est une science récente, qui ne s'appuie que sur un très petit nombre de lacs étudiés scientifiquement. Ces recherches sont en outre ponctuelles, souvent sous forme d'expéditions, en général en saison chaude, et ne donnent que rarement lieu à des séries de mesures en continu à l'intérieur de l'année et sur de longues périodes. Il est donc beaucoup plus difficile d'opérer des classifications lacustres que dans les autres zones.

2 À l'intérieur de la limite de Köppen, le nombre de grands lacs, au sens de C. E. Herdendorf (1990), est faible, puisqu'il n'y a qu'environ 25 plans d'eau de plus de 500 $\mathrm{km}^{2}$, dont 10 de plus de $800 \mathrm{~km}^{2}$ (tableau 1). La plupart se trouvent au nord-est des Territoires du Nord-Ouest et dans l'archipel nord-canadien, quelques-autres au nord de la Sibérie. Certains grands lacs de la planète, pourtant très septentrionaux, sont exclus de ce décompte, puisqu'ils sont cernés de taïga, comme les Grands Lacs de l'Esclave et de l'Ours au Canada ou l'Inari en Finlande. 
Tableau 1 : Les dix plus grands lacs polaires par ordre de superficie décroissante The ten greatest polar lakes in order of surface

\begin{tabular}{|l|c|r|r|c|c|}
\hline \multicolumn{1}{|c|}{ Lac } & Superficie $\left(\mathrm{km}^{2}\right)$ & Altitude $(\mathrm{m})$ & Latitude & Pays & Région \\
\hline Nettilling & 5542 & 30 & $66^{\circ} 30^{\prime} \mathrm{N}$ & Canada & Terre de Baffin \\
Taïmyr & 4650 & 3 & $74^{\circ} 30^{\prime} \mathrm{N}$ & Russie & Péninsule de Taïmyr \\
Dubawnt & 3833 & 236 & $63^{\circ} 08^{\prime} \mathrm{N}$ & Canada & NE NWT \\
Amadjuak & 3115 & 113 & $65^{\circ} 00^{\prime} \mathrm{N}$ & Canada & Terre de Baffin \\
Baker & 1887 & 2 & $64^{\circ} 10^{\prime} \mathrm{N}$ & Canada & NE NWT \\
Yathkyed & 1449 & 140 & $62^{\circ} 41^{\prime} \mathrm{N}$ & Canada & NE NWT \\
Aberdeen & 1100 & 80 & $64^{\circ} 27^{\prime} \mathrm{N}$ & Canada & NE NWT \\
Garry & 976 & 148 & $66^{\circ} 00^{\prime} \mathrm{N}$ & Canada & NE NWT \\
Khantaï & 880 & 73 & $68^{\circ} 20^{\prime} \mathrm{N}$ & Russie & Plateau de Poutorana \\
Teshekpuk & 820 & 2 & $70^{\circ} 35^{\prime} \mathrm{N}$ & E-U (Alaska) & région de Barrow \\
\hline
\end{tabular}

NE NWT : partie continentale du nord-est des Territoires du Nord-Ouest.

3 Ces grands lacs ont pour la plupart été découverts seulement au XIX ${ }^{e}$ siècle mais certains explorateurs ont, dès l'origine, effectué quelques études scientifiques, comme A. F. Middendorf dans les années 1840 pour le Taïmyr ou A. Greely dans les années 1880 pour le Hazen. Les articles pionniers furent ceux du Danois F. S. Andersen (1946) sur les lacs groenlandais, du Soviétique V. N. Greze (1947) sur le Taïmyr et de l'Américain D. S. Rawson (1953) sur les lacs polaires alaskiens et canadiens. Mais les recherches se focalisèrent ensuite sur des plans d'eau de petite taille, dans lesquels les mesures ponctuelles étaient plus représentatives et qui pouvaient conduire à une modélisation moins complexe à réaliser. Les petits lacs orientés et les étangs périglaciaires forment finalement l'essentiel de la bibliographie, les grands lacs étant quelque peu délaissés.

Peut-on tenter une synthèse bibliographique de l'ensemble des lacs polaires, petits et grands, pelliculaires et profonds? Les typologies thermiques et mictiques des lacs de la planète, les seules à faire intervenir une classe polaire, sont-elles suffisantes pour déterminer les spécificités des lacs polaires? Quels sont les critères les plus pertinents pour regrouper les lacs polaires?

\section{Les critères de classification des lacs polaires}

5 Parmi les grands manuels de limnologie à l'échelle planétaire, seul celui de M. J. Burgis et $\mathrm{P}$. Morris (1987) tente une typologie zonale, dans laquelle les lacs polaires forment une classe commune avec les lacs de montagne, fondée sur l'influence de la froideur de l'eau sur le cycle trophique. Chez les auteurs ayant créé une classification des températures de l'eau et des modes de brassage des lacs à l'échelle mondiale, les lacs polaires formaient d'abord une classe entière (Forel, 1895), puis, devant la complexité croissante des organigrammes, se sont retrouvés écartelés en plusieurs groupes (Hutchinson et Löffler, 1956 ; Lewis, 1983 ; Touchart, 2002). Le nombre de critères pris en compte pour définir l'originalité des plans d'eau polaires est plus grand chez J. E. Hobbie (1980), mais seuls les organismes pelliculaires, essentiellement les étangs périglaciaires, sont concernés et aucun sous-type n'est proposé. Il convient donc de poser succinctement quelques jalons d'une réflexion plus globale.

6 Les lacs de la zone polaire sont pour la plupart des lacs de modelé et ont en ce sens une origine zonale. Ce sont soit des lacs dus à un héritage glaciaire, de surcreusement ou d'accumulation morainique, soit des lacs dus à un processus morphodynamique actuel, glaciaire (par exemple les lacs d'obturation glaciaire) ou périglaciaire (par exemple les 
lacs d'alass dus à la fusion d'un volume de pergélisol). Cette origine fréquente leur donne une parenté commune de lacs récents, ayant laissé peu de temps à l'évolution biogéographique et sans formation d'endémisme biologique. Cependant, les héritages glaciaires, y compris d'inlandsis, débordent très largement le monde polaire et l'essentiel des lacs de cette origine se trouvent en fait aujourd'hui en zone tempérée. La difficulté de prise en compte du critère de l'origine morphologique de la cuvette lacustre est encore beaucoup plus grande dans le cas des plans d'eau comme le Taïmyr, qui prend place dans un fossé d'effondrement (Létolle et Touchart, 1998). Cette origine structurale se suffit à elle-même sans intervention morphoclimatique et est par essence azonale. En fait, la pertinence de ce critère typologique diminue proportionnellement avec l'augmentation de la taille du lac.

7 Le système morphogénique polaire du bassin versant, qui conduit au comblement progressif du lac, rassemble un certain nombre de processus communs liés au froid, distinguant surtout deux types de plans d'eau. Les uns se trouvent en domaine glaciaire, les autres, plus nombreux, sont alimentés en conditions périglaciaires, où l'alternance du gel et du dégel est majeure dans la fourniture de sédiments et les pulsations d'avancée des deltas lacustres (Axelsson, 1967). Mais ce critère typologique, comme le précédent, perd de son acuité pour les grands lacs, qui acquièrent une certaine indépendance face à l'influence de leur bassin d'alimentation. Ils entretiennent une érosion littorale propre et assurent une redistribution autonome de leurs sédiments selon des modalités glacielles qui ne suivent pas les mêmes rythmes que ceux du bassin. L'action littorale du pied ${ }^{1}$ de glace lacustre ou encore la prise en glace du centre des grands lacs profonds subissent des décalages temporels parfois très grands avec l'évolution des tributaires, voire, par le dépassement de certains seuils, permettent un changement radical de modalité, qui existe aussi dans les grands lacs de milieu tempéré continental (Rogozin, 1993).

8 Hors quelques exceptions de lacs de l'Antarctique dont la salinité est due à la forte évaporation dans certains terrains déglacés (Burgis et Morris, 1987), les lacs polaires sont presque toujours oligotrophes, possédant peu de nutriments et d'ailleurs, plus généralement, peu d'éléments dissous. Mais cette faiblesse existe aussi dans d'autres conditions, en particulier dans les hautes montagnes tempérées, et, quoi qu'il en soit, la variété lithologique des bassins est un élément déterminant qui possède des caractères azonaux. En fait, la faiblesse de la production biologique primaire est surtout liée à la froideur de l'eau.

9 L'hydrologie des lacs polaires est caractérisée par la permanence du froid de l'eau et une stratification inverse durable, voire pérenne, conduisant au fait que les masses d'eau les plus froides sont au sommet de la colonne d'eau et les moins froides au fond. La grande question typologique, depuis une centaine d'années, se trouve être la très grande fragilité de la stratification de la saison chaude. À partir de la création de la première typologie thermique des lacs par F.-A. Forel (1895), le seuil de $4{ }^{\circ} \mathrm{C}$, celui de la température de densité maximale de l'eau douce, est devenu le paramètre déterminant les types de lacs. L'une des trois classes du limnologue suisse était certes celle des lacs polaires, définis comme étant ceux en stratification inverse en saison froide et en homothermie en saison chaude, sans que la température du lac ne dépasse jamais $4{ }^{\circ} \mathrm{C}$, mais le chercheur helvète, intéressé aux seuls lacs tempérés, avait créé ce groupe d'une manière théorique, sans qu'aucune observation ni mesure réelle n'eût été réalisée pour la corroborer. Ce fut le scientifique russe A. I. Vojeikov (1903) qui s'occupa ensuite de 
caractériser en détail et avec des exemples concrets la famille des lacs polaires, mais sans soulever le problème des modalités complexes du brassage.

Sous son nouveau nom scientifique de micticité, la question des rythmes de brassage fut mise en avant par G. E. Hutchinson et H. Löffler (1956), mais les deux limnologues souhaitaient surtout apporter des nouveautés pour les lacs de montagne et ceux des marges tropicales. Concernant les lacs polaires, ils créèrent cependant la nouvelle classe des lacs amictiques, dans lesquels la couverture glacielle et la stratification inverse, toutes deux permanentes, empêchaient tout brassage. Ils gardaient la vieille famille des lacs polaires au sens de F.-A. Forel (1895) en la renommant celle des lacs monomictiques froids, c'est-à-dire avec une saison de brassage estival chaque année. La zone polaire s'enrichissait donc de deux types de lacs, mais l'appréhension de la saison chaude restait toujours aussi simple.

11 Pourtant, le limnologue japonais S. Yoshimura avait pressenti cette complexité dès 1936 et créé à ce sujet la classe des lacs subpolaires. En effet, les lacs inversement stratifiés pendant l'essentiel de l'année ne sont-ils pas susceptibles de connaître des homothermies à plus de $4^{\circ} \mathrm{C}$ en été, construites par des alternances fréquentes de stratifications directes très fragiles et de multiples déstratifications ? Postérieurement à G. E. Hutchinson et H. Löffler (1956), qui n'en avaient pas tenu compte, d'autres auteurs furent sensibles à cette nuance à propos de lacs de la marge polaire d'Amérique du Nord (Berg, 1963).

12 Finalement, le bouleversement typologique fut celui proposé par W. M. Lewis (1983), s'appuyant sur le fait que, vu la faiblesse d'une stratification thermique inverse en terme de densité, l'important se trouve être de différencier les stratifications inverses avec prise en glace, où une vraie barrière au mélange de l'eau existe, des stratifications inverses sans prise en glace, qui sont presque des homothermies potentielles. En d'autres termes, dans le fonctionnement réel du brassage des lacs d'eau douce, le seuil de prise en glace n'est-il pas plus important que celui de la température de densité maximale? Cette question iconoclaste, qui lézardait les fondements de 88 ans de typologies mondiales reposant sur le passage ou non à travers la température de $4{ }^{\circ} \mathrm{C}$, prenait une importance cruciale pour les lacs polaires.

13 En effet, la définition des lacs monomictiques froids est de ne jamais posséder une eau dépassant $4{ }^{\circ} \mathrm{C}$, d'être inversement stratifiés en hiver et brassés en été. Certes, ces lacs existent sans doute, mais il se trouve que les mesures de terrain en milieu polaire n'en détectent jamais. À l'inverse, il existe assurément d'autres lacs polaires, les plus nombreux, qui, leur ressemblant par leur stratification inverse l'essentiel de l'année, connaissent cependant un été suffisamment chaud pour dépasser la température de $4{ }^{\circ} \mathrm{C}$, sans qu'il le soit assez pour provoquer une stratification directe durable. Celle-ci reste toujours très fragile et la fréquence de la disparition de la thermocline provoque de fréquents brassages pendant la saison chaude. Cela pose la question de la différence entre les lacs profonds de milieu polaire, dont certains peuvent éventuellement être vraiment monomictiques froids (Schindler et al., 1974), et la multitude de plans d'eau pelliculaires de cette zone hydroclimatique, qui sont polymictiques froids, continus ou discontinus (Lewis, 1983).

Nous avons récemment apporté à la classification thermo-mictique des lacs la nouvelle notion d'irrégularité interannuelle (Touchart, 2002). Certains lacs polaires peuvent être amictiques certaines années, mais monomictiques froids lors d'autres années. La dimicticité, répandue en zone tempérée, est même tout à fait possible en zone polaire, 
au moins certaines années. Ainsi, le lac Schrader, directement stratifié en été 1958, avec un épilimnion stable à $10^{\circ} \mathrm{C}$, n'a pourtant jamais dépassé $4{ }^{\circ} \mathrm{C}$ lors de l'été suivant (Hobbie, 1984). Ce principe de l'irrégularité, vrai pour le rythme de brassage, l'est aussi pour la prise en glace et il nous semble qu'il s'agit là du critère majeur.

En effet, ce concept permet à la fois de distinguer les lacs polaires par rapport à ceux des autres zones, mais aussi de différencier entre eux les sous-types de lacs polaires. Ce sont tous des lacs couverts, mais la hiérarchie provient de la durée de l'englacement et de l'épaisseur de la glace. Cela est crucial pour l'oxygénation des lacs polaires, donc leur fonctionnement vital. Le lac est une sorte de vase clos quand il est pris en glace, quand les concentrations en oxygène varient sans contact avec l'atmosphère, c'est-à-dire, pour simplifier, qu'il n'y a plus que de la consommation (Welch, 1974). Pendant toute la durée d'existence de cet obstacle, la quantité d'oxygène diminue. Cela peut aller jusqu'à la quasi-disparition de cet élément vital et on comprend alors l'importance du critère de régularité ou non de la fonte de la banquise lacustre d'une année à l'autre. Pourtant, à l'inverse, quand il n'y a pas de barrière de glace, les valeurs absolues d'oxygène atteignent des records dans les lacs polaires, car l'eau, très froide, peut en contenir beaucoup, et les brassages par les vents sont particulièrement efficaces. Cette influence de la durée de l'englacement et de l'épaisseur de la glace, régulière ou non, se retrouve dans la composition de l'eau en éléments dissous, qui est l'autre élément vital du fonctionnement de l'ensemble de la chaîne alimentaire. La prise en glace prélève l'eau tout en laissant les éléments dissous, qui donc se concentrent dans l'eau; à l'inverse, lors de la fonte, la libération d'eau presque distillée dilue les concentrations. Ces deux processus existent déjà dans les lacs à couverture permanente, quand la tranche se contente de changer d'épaisseur, tantôt fondant un peu tantôt croissant un peu, mais ils prennent une importance démesurée dans les lacs à couverture temporaire, causant alors une tension biologique très forte. Certains lacs polaires ont donc une chaîne trophique équilibrée et régulièrement entretenue chaque année

Finalement, la typologie hiérarchisée des lacs polaires proposée cherche à se fonder sur la décroissance des contraintes limnologiques pour la vie, en emboîtant les critères suivants: 1) Permanence ou non de la couverture de glace sur au moins un cycle trophique annuel; 2) type et épaisseur de la glace; 3) régularité ou non de la couverture de glace ; 4) taux de couverture ; 5) profondeur du lac.

\section{Les lacs à couverture de glace permanente}

17 L'obstacle le plus important, en terme d'épaisseur et de durée, qui puisse exister entre les lacs et l'atmosphère est représenté par un glacier continental. Ces lacs sousglaciaires connaissent un isolement absolu sous les inlandsis, mais des vidanges vers l'extérieur peuvent se produire sous les calottes. Dans le cas d'une couverture de glace lacustre, la barrière est beaucoup plus fragile. Pour ces lacs sous-glaciels, la notion de permanence de la glace est alors à discuter.

\section{Les lacs sous-glaciaires}

Plusieurs lacs infra-glaciaires, parfois de grande taille, ont été découverts sous l'inlandsis antarctique ces dernières décennies. Les épaisseurs de glace peuvent alors atteindre plusieurs milliers de mètres et l'ancienneté de cette barrière peut se compter 
en dizaines de milliers d'années. Ces lacs peuvent se former et subsister, sous 3000 à $4000 \mathrm{~m}$ de glace servant d'isolant thermique, grâce au flux géothermique (Oswald et Robin, 1973). Il en existe une centaine (Giles, 2004), parmi lesquels le plus grand se trouve être celui de Vostok (Petit, 1998), qui s'étend sur près de $10000 \mathrm{~km}^{2}$ et possède une tranche d'eau de $1200 \mathrm{~m}$. Du fait de la pression de la glace, l'eau se trouve sous forme liquide, bien qu'elle soit à une température de $-2,5^{\circ} \mathrm{C}$, et sa concentration en oxygène est si élevée qu'elle interdit toute vie. Les traces d'ADN retrouvées dans la glace d'eau de lac regelée proviendraient de bactéries de source chaude, sans doute issues de fissures profondes du socle et expulsées lors de séismes pour venir mourir dans le plan d'eau (Bulat et al., 2004).

Sous les calottes glaciaires, de taille moindre, un exutoire peut être trouvé, au moins temporairement. C'est le cas en Islande, sous le glacier Vatna, où la chaleur du substrat, d'origine volcanique, fait fondre progressivement la glace et se former un plan d'eau infra-glaciaire, le lac de Grim. Quand il est devenu important, il se vide, provoquant ainsi une crue de débâcle, dite jökulhlaup. La périodicité de cette vidange était d'environ dix ans de la fin du Moyen Âge jusqu'à la Seconde Guerre mondiale (Thorarinsson, 1953 ; Lliboutry, 1965), puis un rythme de cinq ans s'est dégagé (Bodéré, 1977) et un jökulhlaup eut encore lieu en 1996 (Godard et André, 1999). Cependant, le volume des vidanges récentes est plus faible que celui des décennies précédentes (Gudmundsson et al., 1995). Des simulations permettent aujourd'hui de tenter de prévoir les cycles approximatifs de ces vidanges lacustres (Björnsson, 1992).

\section{Les lacs sous-glaciels}

20 Les lacs polaires pris par la banquise posent un double problème typologique, l'un temporel, l'autre spatial (tableau 2). Étant entendu que la glace lacustre isole forcément tous les plans d'eau polaires, et même de nombreux lacs de la zone tempérée, pendant une longue saison froide, la question ne se pose que pour la saison chaude. Qu'en est-il de la permanence de la glace et le seuil pertinent est-il celui du dépassement d'une année complète sans fonte ? La notion d'irrégularité interannuelle de la température de l'eau et de la qualité de la glace lacustre doit devenir un critère typologique essentiel (Touchart, 2002), car certains lacs peuvent être englacés lors de certains cycles annuels complets, sans l'être lors de tous les cycles annuels. Quels sont d'autre part les changements induits par une disparition seulement partielle de la couverture dans un plan horizontal, d'autant plus probable que le lac est de grande taille et permet le développement d'une zonation thermique?

Tableau 2 : Typologie des lacs à couverture glacielle et exemples dans le milieu polaire de I'Atlantique Nord Classification of frozen lakes (lacustrine ice cover) and examples in the North-Atlantic polar zone

\begin{tabular}{|c|c|c|}
\hline & Couverture estivale complète & Couverture estivale partielle \\
\hline \begin{tabular}{|l|} 
Couverture pluriannuelle régulière \\
Couverture pluriannuelle irrégulière
\end{tabular} & $\begin{array}{l}\text { Lac Angiussaq } \\
\text { Anneks Sø }\end{array}$ & $\begin{array}{l}\text { Lac Hazen } \\
\text { Lac Char }\end{array}$ \\
\hline
\end{tabular}

Dans l'ordre décroissant de l'importance de l'obstacle glaciel, les lacs les plus isolés sont ceux à couverture estivale complète pluriannuelle régulière. Jamais la glace n'a fondu au point que l'eau soit en contact avec l'air, même dans une partie réduite du lac. Au nord-ouest du Groenland, le lac Angiussaq, profond de $187 \mathrm{~m}$, est le principal de ces 
plans d'eau. Sa banquise pérenne atteint 3,4 $\mathrm{m}$ en saison froide et reste épaisse de 1,4 $\mathrm{m}$ en saison chaude; elle se raccorde au barrage glaciaire qui ferme le lac et en est à l'origine (Barnes, 1960). Il n'existe sans doute pas plus de cinq autres lacs importants de ce type en Arctique (Hobbie, 1984), dont la couverture glacielle ne dépasse en général pas 2,5 m pendant la saison la plus froide (Burgis et Morris, 1987). En Antarctique, la banquise pérenne des lacs peut atteindre $4 \mathrm{~m}$, voire approcher exceptionnellement $6 \mathrm{~m}$ (Heywood, 1972). En saison chaude, l'épaisseur gelée peut perdre $1 \mathrm{~m}$, comme sur les lacs Vanda $\left(77^{\circ} \mathrm{S}-161^{\circ} \mathrm{E}\right)$ et Fryxell $\left(77^{\circ} \mathrm{S}-163^{\circ} \mathrm{E}\right)$, et l'eau être réchauffée par les radiations traversant la glace (McKay et al., 1985), tout en restant en stratification inverse (Vincent, 1981). Le flux thermique estival a l'avantage d'être caractérisé par sa constance (Wetzel, 2001), qui ne peut cependant pas obvier à l'inconvénient de sa faiblesse, si bien que la vie est très réduite. Dans le Vanda, la production de carbone est de 7,2 $\mathrm{mg}$ par $\mathrm{m}^{3}$ par jour à $25 \mathrm{~m}$ de profondeur et $0,2 \mathrm{mg}$ à $60 \mathrm{~m}$ (Goldman et al., 1967) et, si l'on exclut les protozoaires, le zooplancton est totalement absent (Hobbie, 1984). Mais la vie parvient à se développer à l'intérieur même de la couche de glace, grâce à la fonte de poches d'eau dans la banquise lacustre et aux apports nutritifs des sédiments d'origine éolienne interstratifiés avec la glace. Un cycle de photosynthèse, de fixation de l'azote et de décomposition a ainsi été étudié en Antarctique, à l'intérieur de la couverture glacielle pérenne du lac Bonney (Priscu et al., 1998).

Le deuxième type est celui des lacs dont la couverture estivale n'est complète que certaines années, montrant ainsi une irrégularité. Certains étés, aucune partie du lac n'est en contact avec l'air, lors d'autres étés quelques portions du lac sont libérés des glaces, mais jamais le lac n'est entièrement libre de glace. Ce sont les années les plus neigeuses qui empêchent une grande épaisseur de glace et provoquent sa disparition partielle lors de la saison chaude. D'après les descriptions de A. Trolle (1913), l'Anneks Sø du Nord-Est du Groenland se conduit ainsi.

Le troisième cas est celui des lacs dont la permanence des glaces estivales est celle d'une couverture partielle. Jamais la glace n'a fondu sur la totalité du lac, mais elle libère chaque été certaines portions du plan d'eau. Au nord de l'île d'Ellesmere, le lac Hazen $\left(82^{\circ} \mathrm{N}, 70^{\circ} \mathrm{O}\right)$ appartient à ce type. S'étendant sur $542 \mathrm{~km}^{2}$ pour une profondeur maximale de $280 \mathrm{~m}$, c'est le grand lac le plus septentrional du monde. Présenté tantôt comme amictique (Herdendorf, 1990), tantôt comme monomictique froid (Hutchinson, 1957), il se distingue plutôt par la régularité interannuelle de sa fonte partielle. Grâce à cette qualité, la production primaire, tout en restant très faible, atteint $39 \mathrm{mg}$ de carbone par $\mathrm{m}^{3}$ par jour en août et un zooplancton formé de cyclopes et de daphnies se développe (Saville, 1964). L'ombre de l'Arctique (Salvelinus alpinus), à croissance très lente, est le seul poisson présent dans le Hazen (McLaren, 1964).

24 L'irrégularité d'une couverture estivale partielle forme un dernier cas un peu plus complexe, qui assure la transition avec les lacs à couverture de glace temporaire. Lors de la plupart des étés, ces lacs ne sont que partiellement libérés par les glaces. Mais il existe certaines années pendant lesquelles le lac est entièrement libre, et, à l'inverse, d'autres étés, plus rares, pendant lesquels la banquise reste complète. Il en est ainsi,

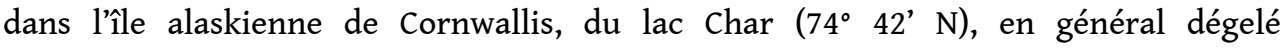
partiellement ou complètement 5 à 6 semaines par an avec une température de surface atteignant 4 à $5{ }^{\circ} \mathrm{C}$, mais qui a connu certains cycles annuels avec une couverture totale (Schindler et al., 1974). Du fait des fortes variations du tapis neigeux recouvrant la glace, les situations montagnardes aident à cette irrégularité, rencontrée par J. E. 
Hobbie (1973) dans la chaîne de Brooks à propos du lac Peters. L'ichtyofaune est parfois plus diversifiée que dans les types de lac précédents. L'ombre de l'Arctique continue cependant de dominer, au point que, appelé «char » par les Anglo-Saxons, il a donné son nom au lac américain homonyme.

\section{Les lacs à couverture de glace temporaire}

Les lacs à couverture temporaire, pris par une banquise saisonnière d'un à deux mètres d'épaisseur, ont la particularité d'être totalement libres de glace pendant plusieurs semaines chaque année. Subissant un très fort marnage, ils sont susceptibles de disparaître en saison froide si l'épaisseur de leur tranche d'eau est insuffisante. Le critère typologique majeur est donc fonction de la proportion de l'épaisseur de glace par rapport à la tranche d'eau totale.

\section{Les plans d'eau pelliculaires périglaciaires}

Les plans d'eau superficiels sont très nombreux en conditions périglaciaires, où ils sont construits par les processus cryokarstiques. Ceux de moins de $2 \mathrm{~m}$ de profondeur sont susceptibles de geler complètement pendant la saison froide, de la surface au fond, disparaissant ainsi saisonnièrement. Ce sont en fait des étangs temporaires, que d'aucuns appellent abusivement des lacs. Ils ont été largement étudiés en Alaska, notamment dans la région de Barrow. Ces plans d'eau, mais aussi de véritables lacs peu profonds, où quelques poches d'eau de fond stagnante subsistent sous 1 à $2 \mathrm{~m}$ de glace, ont de nombreux points communs dus à leur caractère pelliculaire.

En milieu polaire, les étangs et les lacs superficiels gèlent très tôt, souvent dès septembre, du fait de l'absence d'inertie calorifique elle-même causée par leur faible volume. Ils connaissent des différences de concentration en éléments dissous extrêmement marquées (Howard et Prescott, 1973), d'où une très forte tension biologique. Il en résulte une faible quantité de phytoplancton et un nombre d'espèces réduit, conduisant à la domination quasi-exclusive des cellules de très petite taille, sous forme de nanoplancton (Prescott, 1953). Les poissons manquent le plus souvent, si bien que, du fait de cette absence de prédateur, le zooplancton se développe, tout particulièrement les micro-crustacés, qui forment ainsi l'essentiel des espèces (Stross et al., 1980). La plupart de ces espèces zooplanctoniques sont malgré tout forcées de dégager une longue phase de dormance. Les contraintes restent donc très prononcées malgré le caractère saisonnier de la prise en glace. Seule une profondeur lacustre plus marquée peut, à conditions climatiques égales, les atténuer.

\section{Les lacs profonds à couverture glacielle saisonnière}

Dans les lacs profonds du milieu polaire à gel saisonnier, la dimicticité est de règle, si bien que le rythme de brassage et le comportement trophique se rapprochent de ceux des lacs du domaine continental de la zone tempérée. La couverture glacielle est cependant plus longue, durant fréquemment 8 à 9 mois, et souvent plus épaisse, entre 1 et $2 \mathrm{~m}$. Ce rythme saisonnier s'accompagne de variations de niveau très amples, qui ajoutent d'importantes contraintes à la vie mais n'atteignent pas l'acuité des problèmes 
d'alternance entre l'apparition et la disparition du plan d'eau lui-même tels qu'ils existaient chez les lacs pelliculaires.

29 En effet, à cause de la libération des calories emmagasinées dans un gros volume d'eau, les lacs profonds gèlent plus tard que les lacs superficiels des mêmes régions. Ils alimentent, par de l'eau sous-glacielle, des émissaires fluviaux qui sont, parmi tous les cours d'eau polaires, ceux s'écoulant le plus tard dans la saison. Cela a pour conséquence de vider progressivement les lacs, qui ne sont plus alimentés par leurs affluents, déjà gelés. De très bas niveaux se mettent alors en place, qui contrastent avec les énormes apports par débâcle dus à la fonte dans le bassin au début de la saison chaude. Le marnage acquiert donc une importance considérable dans le fonctionnement de ces lacs. Le niveau du lac Taïmyr augmente ainsi de 6 à $7 \mathrm{~m}$ entre mai et juillet (Greze, 1947), pendant que le débit moyen mensuel de son émissaire est multiplié par plus de 2000 (Doronina et Sisko, 1970). Ces variations verticales s'accompagnent d'une augmentation de la superficie lacustre de 1200 à $4650 \mathrm{~km}^{2}$ en quelques semaines, car, en dehors de la cuvette centrale, profonde de $26 \mathrm{~m}$, de larges baies sont en pente douce. Dans les lacs de surcreusement glaciaire, très encaissés, le fort marnage ne provoque pas de grandes variations de superficie. C'est le cas des lacs noriliens du plateau de Poutorana, comme le Lama ( $466 \mathrm{~km}^{2}, 254 \mathrm{~m}$ de profondeur) et le Gloubokoïé (136 km², $185 \mathrm{~m}$ de profondeur), dont les variations de niveau atteignent près de $4 \mathrm{~m}$ chaque année (Bogdanov, 1985). Le marnage des lacs polaires d'Amérique du Nord est plus faible que celui des lacs sibériens, tournant autour du mètre, cas du plus vaste lac polaire de la planète, le Nettilling (Oliver, 1964).

Malgré ce fort marnage et l'épaisseur de la banquise saisonnière, il reste toute l'année une colonne d'eau importante dans les lacs profonds, permettant une vie plus riche que dans les plans d'eau superficiels. Le phytoplancton commence à se développer sous la glace avant la fusion (Hobbie, 1964) et peut même atteindre son maximum avant que la couverture glacielle ait disparu (Kalff et al., 1972). Il existe cependant de grandes différences selon la transparence de la glace et surtout, l'importance ou non de la neige posée sur elle. S'il y a peu de neige, la lumière peut traverser la glace et provoquer une explosion planctonique précoce, parfois dès février. Si le lac est de grandes dimensions, la zonation géographique dépend en grande partie du vent, qui accumule la neige par endroit et diminue la production primaire, mais la balaie sur d'autres parties du plan d'eau gelé et accroît les possibilités trophiques.

Les contraintes polaires restent pourtant fortes. La froideur de l'eau provoque une telle lenteur des taux de décomposition que les nutriments sont immobilisés dans la matière organique morte. La chaîne trophique a du mal à se développer complètement et la manifestation principale en est la faiblesse du monde animal et la quasi-absence de certains groupes, comme les éponges et les amphibiens. Les poissons, représentés par un faible nombre d'espèces, ont une croissance lente, mais ces lacs oligotrophes ont malgré tout une forte potentialité d'exploitation halieutique, car le peuplement est essentiellement constitué de salmonidés, le plus souvent d'ombres de l'Arctique. Les grands lacs polaires les plus anciens, qui ont eu le temps de développer des formes d'endémisme, sont les plus riches en nombre d'espèces (Pavlov et Savvaitova, 1999), surtout si d'anciennes communications avec la mer ont permis une évolution complexe à travers les pulsations glacio-isostatiques (Touchart, 2000). durée de la prise en glace est moindre, où la valeur de $10^{\circ} \mathrm{C}$ est dépassée dans 
l'atmosphère pendant un ou deux mois et permet une stratification directe des masses d'eau lacustres pendant quelques semaines. Dans la péninsule de Kola, au sud de Mourmansk, le lac Imandra, qui s'étend sur $880 \mathrm{~km}^{2}$ et a une profondeur de $67 \mathrm{~m}$, en est un bon exemple. Son bassin est presque uniquement couvert de toundra de montagne, mais une taïga chétive s'approche des littoraux, grâce à des températures de l'air de 12,9 et $10,7^{\circ} \mathrm{C}$ en juillet et août (station de Kirovsk). Sur près de 80 ans d'observation, la prise en glace a duré au minimum 173 jours et au maximum 226 jours. Une stratification directe s'établit de juin à octobre et le marnage n'est que de $1 \mathrm{~m}$ (Moisenko et Sandimirov, 2002). Le cycle trophique tend alors à se rapprocher de celui des grands lacs dimictiques du domaine continental de la zone tempérée, pour former le grand groupe des lacs « tempérés-froids » de I. V. Baranov (1962).

\section{Conclusion}

L'étude scientifique des plans d'eau polaires privilégie classiquement les étangs et les plus petits lacs, du fait que la limnologie a une propension à modéliser et la géographie physique une disposition à laisser la géomorphologie dominer. Il était intéressant de tenter d'inverser la démarche habituelle en préférant au contraire une approche de géographie limnologique. Le critère de l'origine du lac doit ainsi s'effacer devant d'autres facteurs, en premier lieu ceux de température de l'eau et de brassage. Dans le cas des lacs polaires, la classification thermo-mictique mondiale nécessite un rajeunissement faisant passer la prise en compte de la glace devant celle de la température de densité maximale et insister sur la question de l'irrégularité interannuelle. Une hiérarchie des contraintes trophiques des lacs polaires peut alors être esquissée, depuis les lacs isolés en permanence, par un glacier ou une banquise, jusqu'à ceux dont la barrière glacielle est saisonnière. Chez ces derniers, la taille prend toute son importance, montrant la valeur des grands lacs profonds dans la production d'une richesse halieutique exploitable.

\section{BIBLIOGRAPHIE}

ANDERSEN (F. S.), 1946. - « East Greenland lakes as habitats for chironomid larvae », Medd.

Grønland, n 100 , p. 1-65.

AXELSSON (V.), 1967. - « The Laitaure delta, a study of deltaïc morphology and processes », Geografiska Annaler, vol. 49, n 1, p. 1-127.

BARANOV (I. V.), 1962. - LimnologicŠeskie tipy ozer SSSR, Leningrad, GidrometeorologicŠeskoe Izd., $276 \mathrm{p}$.

BARNES (D. F.), 1960. -An investigation of a perennially frozen lake, Air Force Surveys in Geophysics $n^{\circ}$ 129 ARCRL-TN-60-660, $134 \mathrm{p}$.

BERG (C. O.), 1963. - « Middle Atlantic states », dans FREY (D. G.) (ed.), Limnology in North America

Madison, University of Wisconsin Press, p. 191-237. 
BJÖRNSSON (H.), 1992. - « Jökulhlaups in Iceland : prediction, characteristics and simulation », Annals of Glaciology, $\mathrm{n}^{\circ}$ 16, p. 95-106.

BoDÉRÉ (J.-C.), 1977. - « Les kettles du sud-est de l'Islande », Revue de Géographie Physique et de Géologie Dynamique, vol. 19, n³, p. 259-270.

BOGDANOV (A. L.), 1985. - « Ocenka nekotoryh antropogennyh èffektov v bassejna ozër plato Putorana : istoriâ izucŠeniâ, morfometriâ i gidrologiâ ozër », dans ADAMENKo (V. N.), EGOROV (A. N.) (red.) Geografiâ ozër Tajmyra, Leningrad, Nauka, p. 184-200.

Bulat (S.), Alekhina (I. A.), Blot (M.), Petit (J. R.), Angelis (M. de), Wagenbach (D.), Lipenkov (V. Y.), VASILEVA (L.), WLOCH (R. D.), LUKIN (V. V.), 2004. - « DNA signature of thermophilic bacteria from the aged accretion ice of Lake Vostok : implications for searching life in extreme icy environments ", Journal of Astrobiology, n 3, p. 1-12.

BURGIS (M. J.), MORRIS (P.), 1987. - The Natural History of Lakes, Cambridge University Press, 218 p.

DoRonina (N. A.), SisKo (R. K.), 1970. - « Reki, ozera, bolota », dans SisKo (R. K.) (red.), Tajmyroseverozemel'skâ̂ Oblast' (fiziko-geograficecŠkaâ harakteristika), Leningrad, GidrometeorologicŠeskoe Izd., p. 238-264.

FOREL (F.-A.), 1895. - Le Léman, monographie limnologique, Lausanne, F. Rouge, T. 2, 651 p.

GILES (J.), 2004. - « Russian bid to drill Antarctic lake gets chilly response », Nature, vol. 430, p. 494.

GodARD (A.), ANDrÉ (M.-F.), 1999. - Les milieux polaires, Paris, A. Colin, coll. « U », 454 p.

Goldman (C. R.), MASON (D. T.), HobBiE (J. E.), 1967. - « Two Antarctic desert lakes », Limnology and Oceanography, $\mathrm{n}^{\circ} 12$, p. 295-310.

GREZE (V. N.), 1947. - « Tajmyrskoe ozero », Izvestiâ Vsesoûznogo GeograficŠeskogo ObsŠcŠestva, vol. $79, n^{\circ} 3$, p. 289-302.

GudMUNDSSON (M. T.), BJöRNSSON (H.), PÁLSSON (F.), 1995. - « Changes in jökulhlaup sizes in Grímsvötn, Vatnajökull, Iceland, 1934-1991, deduced from in situ measurements of subglacial lake volume ", Journal of Glaciology, n 41, p. 263-272.

HERDENDORF(C. E.), 1990. - « Distribution of the world's large lakes », dans TILZER (M. M.), SERRUYA (C.) (ed.), Larges lakes, ecological structure and function, Berlin, Springer, p. 3-38.

HEYwood (R. B.), 1972. - « Antarctic limnology », British Antarctic Survey Bulletin, vol. 18, p. 35-65. HoBBIE (J. E.), 1964. - « Carbon-14 measurements of primary production in two Alaskan lakes », Verhandlungen Internationale Vereinigung für theoretische and angewandte Limnologie, vol. 15, p. 360-364.

-, 1973. - « Arctic limnology : a review », dans BRITTON (M. E.) (ed.), Alaskan arctic tundra, Arctic Institute of North America, Technical Paper n² 25, p. 127-168.

-, 1980. - « Introduction and site description », dans HoBBIE (J. E.) (ed.), Limnology of tundra ponds, Barrow, Alaska, Stroudsburg, Dowden Hutchinson and Ross, p. 19-50.

-, 1984. - « Polar Limnology », dans F. B. TAUB (ed.), Lakes and Reservoirs, Amsterdam, Elsevier, Ecosystems of the world $n^{\circ} 23$, p. 63-105.

HowARD (H. H.), PREscott (G. W.), 1973. - « Seasonal variation of chemical parameters in Alaskan tundra lakes », American Midland Naturalist, n 90, p. 154-164. 
HUTCHINSON (G. E.), 1957. - A Treatise on Limnology, New York, John Wiley and Sons, vol. 1, «Geography, physics and chemistry », 1015 p.

HUTCHINSON (G. E.), LÖFFLER (H.), 1956. - « The thermal classification of lakes », Proceedings of the National Academy of Sciences of the United States of America, Washington, $\mathrm{n}^{\circ} 42, \mathrm{p} .84-86$.

KAlfF (J.), WelCh (H. E.), HolmgReN (S. K.), 1972. - « Pigment cycles in two high-arctic Canadian lakes ", Verhandlungen Internationale Vereinigung für theoretische and angewandte Limnologie, vol. 18, p. 250-256.

LÉtolle (R.), Touchart(L.), 1998. - « Les lacs de l'Asie arctique », dans Grands lacs d'Asie, Paris, L'Harmattan, p. 17-31.

LEWIS (W. M. Jr.), 1983. - « A revised classification of lakes based on mixing », Canadian Journal of Fisheries and Aquatic Sciences, vol. 40, p. 1779-1787.

LliboutRY (L.), 1965. - Traité de Glaciologie, Paris, Masson, 2 tomes, 1040 p.

MCKAY (C. P.), CLOW (G. D.), WHARTON (R. A.), SQUYRES (S. W.), 1985. - « Thickness of ice on perennially frozen lakes ", Nature, $\mathrm{n}^{\circ} 313$, p. 561-562.

MCLAREN (I. A.), 1964. - « Zooplankton of Lake Hazen, Ellesmere Island, and a nearby pond with special reference to the copepod Cyclops scutifer Sars ", Canadian Journal of Zoology, vol. 42, p. 613-629.

MoISENKo (T. I.), SANDiMIRov (S. S.), 2002. - « Fiziko-geograficŠeskaâ xarakteristika rajona », dans MoISENKo (T. I.) (red.), Antropogennye modifikacii èkosistemy ozera Imandra, Moskva, Nauka, p. 6-23. OLIVER (D. R.), 1964. - « A limnological investigation of a large arctic lake, Nettilling Lake, Baffin Island », Arctic, vol. 17, p. 69-83.

OswaLd (G. K. A.), RobiN (G. Q.), 1973. - « Lakes beneath the Antarctic Ice Sheet », Nature, $\mathrm{n}^{\circ}$ 245, p. 251-254.

Pavlov (D. S.), SAvvaitova (K. A.), 1999. - Raznoobrazie ryb Tajmyra, Moskva, Nauka, 208 p.

Petit (J.-R.), 1998. - « La glace, le matériau glace et les calottes glaciaires », Géochronique, nº 68, p. 13-14.

PRESCOTT (G. W.), 1953. - « Preliminary notes on the ecology of freshwater algae in the Arctic Slope, Alaska, with descriptions of some new species ", American Midland Naturalist, $\mathrm{n}^{\circ}$ 50, $\mathrm{p}$. 463-473.

Priscu (J. C.), Fritsen (C. H.), Adams (E. E.), Giovannoni (S. J.), Paerl (H. W.), Mckay (C. P.), Doran (P. T.), GoRdon (D. A.), LANOIL (B. D.), PincKney (J. L.), 1998. - « Perennial Antarctic lake ice : an oasis for life in a polar desert ", Science, vol. 280, p. 2095-2098.

RAWSON (D. S.), 1953.- « Limnology in the North American Arctic and Subarctic », Arctic, vol. 6, p. 198-204.

RoGozin (A. A.), 1993. - Beregovaâ zona Bajkala i Hubsugula, Novosibirsk, Nauka, 168 p.

SAVILLE (D. B.), 1964. - « General ecology and vascular plants of the Hazen Camp area », Arctic, vol. 17, p. 237-255.

SChinder (D. W.), Welch (H. E.), KALfF (J.), BRUnSKILl (G. J.), KRITSCH (N.), 1974. - « Physical and chemical limnology of Char Lake, Cornwallis Island ( $75^{\circ} \mathrm{N}$ lat.) », Journal of Fishery Research Board Canada, vol. 31, p. 585-607. 
Stross (R. G.), Miller (E. C.), Daley (R. J.), 1980. - « Zooplankton », dans Hobbie (J. E.) (ed.),

Limnology of tundra ponds, Barrow, Alaska, Stroudsburg, Dowden Hutchinson and Ross, p. 251-296.

THORARINSSON (S.), 1953. - « Some New Aspects of the Grimsvötn Problem », The Journal of

Glaciology, n 2, p. 267-275.

TOUCHART (L.), 2000. - Les lacs, origine et morphologie, Paris, L'Harmattan, 210 p.

-, 2002. - Limnologie physique et dynamique, une géographie des lacs et des étangs, Paris, L'Harmattan, $395 \mathrm{p}$.

Trolle (A.), 1913. - « Hydrographical observations from the Denmark expedition », Medd.

Grønland, n 41, p. 271-426.

VINCENT (W. F.), 1981. - « Production strategies in Antarctic inland waters : phytoplankton ecophysiology in a permanently ice-covered lake », Ecology, vol. 62, p. 1215-1224.

VojeiKov (A. I.), 1903. - « Les lacs de type polaire et les conditions de leur existence ", Archives des Sciences physiques et naturelles de Genève, vol. 16, p. 300-309.

WeLCH (H. E.), 1974. - « Metabolic rates of arctic lakes », Limnology and Oceanography, vol. 19, p. 65-73.

Wetzel(R. G.), 2001. - Limnology : lake and river ecosystems, San Diego, Academic Press, 1006 p.

YoshimuRa (S.), 1936. - « A contribution to the knowledge of deep water temperatures of Japanese lakes ", Japanese Journal of Astronomy and Geophysics, part I, vol. 13, p. 61-120, part II, vol. 14, p. 57-83.

\section{NOTES}

1. Banquette côtière formée par l'eau du lac gelée sur le rivage et les embruns gelés, le tout surmonté de congères.

\section{RÉSUMÉS}

Le critère typologique de l'isolement dû à la glace est majeur et son importance est replacée en fonction de la régularité interannuelle du taux de la couverture glaciaire ou glacielle. La barrière la plus hermétique est celle d'un glacier continental, suivie par celle d'une banquise lacustre permanente. Chez les lacs à couverture glacielle saisonnière, les plans d'eau superficiels, que le gel est susceptible de faire disparaître chaque année, ont une chaîne trophique beaucoup plus pauvre que les grands lacs profonds, capables de fournir des conditions favorables à la vie malgré l'ampleur du marnage.

The major typological criterion for classifying polar lakes is the isolation by the ice cover. The significance of this phenomenon depends on the inter-annual regularity of the ice regime. Continental glaciers are the main obstacle between water and atmosphere; perennially frozen lakes are largely isolated too. The seasonally frozen lakes are either shallow and deep lakes. Polar 
ponds and shallow lakes, which may completely freeze and disappear each year, are biologically poor, whereas deep polar lakes present a complete trophic cycle despite the great water level fluctuations.

INDEX

Mots-clés : étang, lac, limnologie polaire, micticité, oligotrophie

Keywords : lake, mixing, oligotrophication, polar limnology, pond

\section{AUTEUR}

\section{LAURENT TOUCHART}

Université de Limoges, GÉOLAB, UMR 6042 - CNRS, touchart@unilim.fr 